\title{
MINUTES OF 1989 ANNUAL GENERAL MEETING
}

\section{CANADIAN ASSOCIATION OF TEACHERS OF TECHNICAL WRITING}

(CATTW/ACPRTS)

Sunday May 28, 1989

1. The President called the meeting to order at 16:50. Fourteen CATTW members were present.

2. The members approved the minutes of the 1988 AGM and the agenda for the 1989, inserting one additional item: formation of a Society Newsletter.

3. The President (Jacqueline Bossé-Andrieu):

- Thanked the executive committee for their efforts over the past twelve months, and the programme committee for orchestrating an excellent conference.

- Expressed CATTW's thanks to SSHRCC for their support.

- Gave a special vote of thanks to Christine Parkin for editing a special issue of the Society journal Technostyle.

- Proposed that CATTW continue the study into the technical writing courses and programmes taught across Canada, with a view to developing a definitive list of who is teaching what, what is being taught, and where it is being taught.

The members endorsed Jacqueline's proposal and suggested setting up a committee (of Lil Rodman, Louise Larivière, and Hyacinth Harewood) to carry out the research (which could become the focus of a paper to be presented at next year's conference). Lilita Rodman will investigate whether B.C.'s survey of programmes can be used as a basis for CATTW's study. 
3. The Vice-President (Michael Jordan) expressed his concern that the Society was not attracting enough community college teachers of technical writing to become members or attend conferences. Notices announcing the 1989 conference were sent to all community colleges in Ontario and Quebec, but CATTW received no response. A lengthy discussion ensued, during which:

- The members recommended instituting a membership drive and encouraging "new faces" to be presenters of papers at future conferences. They also accepted Ron Blicq's recommendation that each CATTW member personally contact between three and five potential CATTW members who reside in his or her area of Canada, and urge them to join the Society. To help them, Ron will prepare a draft brochure describing the advantages of belonging to CATTW and, after it has been approved by the executive committee, will arrange to have it printed at no expense to the Society.

- Life member and past president Joan Pavelich suggested appointing two "scoundrels" to urge members to chase membership, and named the two past presidents to fulfill the role of stirrers of interest.

- Michael Jordan recommended, and the members endorsed, including abstracts of papers with the conference announcement and preliminary programme, to encourage teachers to attend and help them convince their administrations of the value of the conference.

Michael Jordan also reported that the executive committee had discussed themes for the 1990 conference, and had narrowed the choice to one of:

"Scientific Writing: Research and Practice."

"Multi-media Technology: Its Effects on Technical Communication."

5. The Immediate Past President (Ron Blicq):

- Congratulated the committees on another excellent year for CATTW. 
Recommended that the Society focus on new developments in communications technology, since teachers of technical writing will have to be ready to teach techniques (such as desktop publishing and preparing computer documentation) that their students will encounter after they graduate.

6. The Secretary-Treasurer (Juanita Giesbrecht--represented by Ron Blicq) reported that the Society has a bank balance of approximately $\$ 2300$, with outstanding invoices of approximately $\$ 500$ yet to be paid. Only 25 members have paid their dues so far for 1989.

Juanita also recommended that each committee chairperson bring a written report to the next AGM. Doing so will simplify minute-taking and, since the reports can be attached to the minutes, shorten the minutes.

7. The Editor of Technostyle (Jennifer Connor) reported that:

- Three issues of the Society journal were issued in 1988 and that, with the help of new word-processing equipment, production of the first of the 1989 issues is on schedule.

- The issues have become larger and are costing about $\$ 600$ each to print.

- Next year will be a period of transition, as editorship will be transferred gradually to a new editor whose term will commence June 1, 1990.

The members enthusiastically congratulated Jennifer on the quality of Technostyle.

8. The Secretary Treasurer (RB for Juanita Giesbrecht) conducted the Society elections, and the following persons were elected to the executive for two years (1989-1991):

- President:

- Vice President:

- Secretary-Treasurer:
Michael Jordan

Queen's University

Lilita Rodman

University of British Columbia

Janet Giltrow

Simon Fraser University 
Because vacancies have been created in two other committees, the following persons were also elected to one-year terms:

- Programme Committee: Louise Larivière Joan E. Pavelich

- Nominating Committee: Joan E. Pavelich

The Nominating Committee also proposed, and the members approved, the following amendment to the CATTW Constitution:

That the term of office for the Editor of Technostyle be reduced from five to three years, and that at the end of the third year the incumbent be given the option to continue as Editor for a further two years.

The Nominating Committee also commented that, although the election of the Editor of Technostyle would not occur until the 1990 AGM, to assure a smooth transition Anne Parker (University of Manitoba) has confirmed her willingness to serve as Editor from June $1,1990$.

9. The upcoming Vice-President (Lilita Rodman, who will chair the $\mathbf{1 9 9 0}$ conference programme committee) proposed, and the members approved, that next year's conference be held on Saturday and Sunday May 26 and 27 in Victoria, BC.

10. The members discussed and approved Jacqueline Bossé-Andrieu's proposal that the Society institute a twice-annual Newsletter containing general news of members, and announcements of events and items of interest. Bruce Lundgren (University of Western Ontario) was mentioned as a potential editor, and Jennifer Connor volunteered to broach the idea to him.

11. The President (Jacqueline Bosse-Andrieu) closed the meeting at $17: 55$, and handed over the reins to Michael Jordan, the new President.

Ron Blicq

Scribe

Jacqueline Bossé-Andrieu

President 


\section{PROCES-VERBAL DE L'ASSEMBLEE GENERALE ANNUELLE DE L'ASSOCLATION CANADIENNE DES PROFESSEURS \\ DE REDACTION TECHNIQUE ET SCIENTIFIQUE (ACPRTS) \\ QUI S'EST TENUE LE 28 MAI 1989 \\ A L'UNIVERSITE LAVAL}

1. La présidente ouvre la séance à $16 \mathrm{~h} 50$. Quatorze membres de l'ACPRTS sont présents.

2. L'ordre du jour est approuvé après l'inclusion d'un point supplémentaire: création d'un Bulletin de l'ACPRTS. Le procèsverbal de la dernière réunion (1988) est approuvé.

3. Rapport de la présidente (J. Bossé-Andrieu):

- La présidente remercie le bureau de l'Association pour le travail effectué ces douze derniers mois et le Comité du programme pour la qualité du programme du présent congrès.

- Elle exprime la reconnaissance de l'ACPRTS à l'égard du Conseil de recherches en sciences humaines qui a, de nouveau cette année, accordé son appui à l'Association.

- Elle remercie aussi tout particulièrement Christine Parkin qui s'est chargée de la publication d'un numéro spécial de Technostyle.

- Elle propose que l'ACPRTS poursuive l'enquête sur les cours et les programmes de rédaction donnés au Canada afin d'en élaborer la liste.

La proposition est approuvée. Un comité est chargé de l'enquête (Lil Rodman, Louise Larivière, H. Harewood). Il envisagera la possibilité de présenter une communication sur ce sujet l'an prochain. Lilita Rodman étudiera la possibilité d'utiliser comme point de départ le questionnaire établi par l'Université de Colombie-Britannique qui a déjà mené une enquête de ce genre. 
4. Rapport du vice-président (Michael Jordan):

Le vice-président exprime son inquiétude devant le manque d'intérêt des professeurs de rédaction technique des colléges communautaires à l'égard de l'Association. Des avis ont été envoyés à tous les collèges communautaires de l'Ontario et du Québec pour annoncer le congrès de 1989 , mais en vain.

Au cours d'une longue discussion, plusieurs recommandations sont présentées:

- Les délégués recommandent qu'une campagne de recrutement soit lancée pour augmenter le nombre des membres de l'Association et pour encourager de nouveaux participants à présenter des communications aux prochains congrès. Ils approuvent aussi la recommandation de Ron Blicq qui propose que chaque membre de l'ACPRTS contacte personnellement dans sa région entre trois et cinq membres potentiels et les pousse à adhérer à l'Association. A cette fin, Ron préparera un projet de prospectus décrivant les avantages que présente l'adhésion a l'ACPRTS. Une fois que ce texte aura été approuvé par le Bureau, Ron le fera reproduire gratuitement.

- Joan Pavelich, ancienne présidente et membre à vie de l'Association, suggère que les deux anciens présidents se chargent de prendre les moyens nécessaires pour que les membres participent à la campagne de recrutement et cherchent à éveiller l'intérêt envers l'Association.

Michael Jordan propose que le programme préliminaire et les annonces concernant le prochain congrès soient accompagnés d'un résumé des communications. Cette mesure inciterait les professeurs à assister au congrès et leur permettrait de convaincre leur établissement de l'utilité du congrès. Recommandation approuvée. Michael Jordan signale que le Bureau a discuté de thèmes possibles pour le congrès de 1990. Les deux thèmes suivants ont été retenus:

"La rédaction scientifique: recherches et applications" "Les répercussions des nouveaux moyens de communication sur la rédaction technique" 
5. Rapport de l'ancien président de l'ACPRTS (Ron Blicq):

- Ron Blicq félicite les comités pour le travail effectué depuis l'an dernier.

- Il recommande que l'Association prête une attention particulière aux nouveaux moyens de communication engendrés par la technologie. En effet, les professeurs de rédaction technique doivent être prêts à enseigner de nouvelles techniques (telles que l'éditique et la rédaction de documents d'informatique) dont leurs étudiants pourraient avoir à se servir sur le marché du travail.

6. Rapport de la secrétaire-trésorière (Juanita Giesbrecht):

Au nom de J. Giesbrecht, Ron Blicq présente les états financiers de l'Association: le solde en banque de l'ACPRTS s'élève à environ $2300 \$$ sans compter les quelque $500 \$$ de cotisations dus pour cette année. Jusqu'ici, seulement 25 membres ont versé leur cotisation. Juanita recommande que le président de chaque comité présente un rapport écrit à l'Assemblée générale. De cette façon, la prise de notes serait simplifiée et le procés-verbal raccourci.

7. Rapport de la rédactrice de Technostyle (Jennifer Connor):

La rédactrice de Technostyle signale que:

- Trois numéros de Technostyle ont paru en 1988 et que, grâce à une nouvelle machine de traitement de texte, la production du premier numéro de 1989 est sorti à temps.

- Les numéros sont plus volumineux qu'avant et reviennent a environ $500 \$$ chacun.

- L'an prochain sera, pour la revue, une période de transition puisque le mandat de la nouvelle rédactrice débute le $1^{\text {er }}$ juin 1990.

Les délégués félicitent chaudement Jennifer pour la qualité de Technostyle. 
8. Ron Blicq, en remplacement de la secrétaire-trésorière (Juanita Giesbrecht) préside les élections. Sont élus pour un mandat de deux ans (1989-1991):

- Président: Michael Jordan

Queen's University

- Vice-présidente: Lilita Rodman

Université de Colombie-Britannique

- Secrétaire-trésorière: Janet Giltrow

Simon Fraser University

Du fait de ces nominations, des postes sont vacants dans deux comités; sont done élus pour un mandat d'un an:

- Comité du programme: Louise Larivière Joan E. Pavelich

- Comité des candidatures: Joan E. Pavelich

Le Comité des candidatures propose d'apporter la modification suivante aux statuts de l'ACPRTS:

Le mandat de rédacteur en chef de Technostyle est de trois ans (et non plus de cinq ans): à la fin de la troisième année, il peut être prolongé de deux ans. Proposition approuvée.

Le Comité des candidatures signale en outre que, même si l'élection officielle de la nouvelle rédactrice de Technostyle n'aura lieu qu'au cours de l'Assemblée générale de 1990, Anne Parker (Université du Manitoba) consent à assumer les fonctions de rédactrice dès le premier juin 1990.

9. La prochaine vice-présidente (Lilita Rodman), qui sera responsable du programme du congrès de 1990, propose que l'an prochain le congrés--qui se tiendra à Victoria (C.-B.)--ait lieu les samedi et dimanche 26 et 27 mai. Proposition acceptée.

10. Il est proposé par J. Bossé-Andrieu que l'Association fasse paraitre un bulletin semestriel contenant des nouvelles d'intérêt général, les annonces de congrès, etc. Proposition acceptée. Parmi les noms de 
responsables possibles, celui de Bruce Lundgren (Université de Western Ontario) est avancé. Jennifer Connor lui en parlera.

11. La présidente, J. Bossé-Andrieu, lève la séance à $17 \mathrm{~h} 55$ et remet la présidence à Michael Jordan.

Le Secrétaire de séance,

La Présidente

Ron Blicq

J. Bossé-Andrieu 


\title{
PRELIMINARY PROGRAMME
}

Technical and Scientific Writting and the Impact of New Technologies

CATTW/ACPRTS

\author{
LEARNED SOCIETIES \\ UNIVERSITY OF VICTORIA
}

May 26-27, 1990

\section{SATURDAY, MAY 26}

9:00-10:30

Nancy Carlman and Janice Baker, "Not a Piece of Cake: A Technical Writing Instructor Writes a Technical Report."

Gillian Crowley, "The Fiction of the 'Real' Audience: A Comparison of Audience Perception Experienced by College Students and Business People."

Marcia Macaulay, "New Information in Oral Exposition."

$10: 45-12: 15$

Candace Séguinot, "Identifying Skills in Technical Writers."

Louise Larivière, "Le protocole épistolaire en française et en anglais."

Anne Parker, "Collaborative Writing in the Technical Writing Classroom."

\section{LUNCH}

$1: 15-3: 15$

Elizabeth Shore, "The Rhetoric of Environmental Writing."

Nan Johnson, "Teaching Technical Writing as Rhetoric."

Ron Blicq, "Teaching Technical Writing in Industry."

Christiane Melançon, "Le commerce et l'administration, ou comment enseigner la rédaction technique à de futurs traducteurs."

Amanda Goldrick-Jones and Deborah Scott, "Creating a Province-wide Directory of Communication Courses: Methods and Problems"

Lionel Meney, "D'une difficulté de l'enseignement de la rédaction technique en milieu francophone." 
SUNDAY, MAY 27

$9: 00-10: 30$

Michael Jordan, "Openings in Informal Scientific Prose."

Jacqueline Bossé-Andrieu, "Le recul du français comme langue des sciences."

Mimi Zeiger, "Biomedical Writing: The State of the Art."

$10: 45-12: 00$

Cynthia Cunningham and Kathy Lea, "Bibliographic Instruction as a Means of Improving Scientific Research Paper Quality."

Victoria M. Mikelonis, "Computer-mediated Instruction for Academia and the Professions."

\section{LUNCH}

$1: 15-2: 45$

Barbara McDaniel, "The Changing Role of Writers in Computing Science."

Cheiva Kanaganayakam, "Text and Subtext in Designing Hands-On Course Notes."

Kenneth Hoeppner, "HyperCard as a Medium for Teaching the Writing of Instructions."

3:00-5:00 Ruth Ellen Greenwood, "Combining Teachers, Students, and Computers Creates Learning and Change."

Maria Burgar, "The Short-Term and Long-Term Benefits to be Derived from Introducing a Computer-Infused Teaching Methodology into the Classroom."

Jean-Yves Fréchette, "LogiTexte et la conception textuelle assistée par ordinateur."

Charles Doutrelepont, "Rédaction technique et logiciels de traitment de textes." 